\title{
Four-term leaping recurrence relations
}

\author{
弘前大学大学院理工学研究科 小松 尚夫 (Takao Komatsu) ${ }^{1}$ \\ Graduate School of Science and Technology \\ Hirosaki University
}

\section{Introduction}

Given a three-term linear recurrence relation $Z_{n}=T(n) Z_{n-1}+U(n) Z_{n-2}(n \geq 2)$, where the initial values $Z_{0}, Z_{1}$ are arbitrary integral values, and $(T(n))_{n \geq 0},(U(n))_{n \geq 0}$ are integer sequences with $U(n) \neq 0$ for all $n \geq 0$.

In 2008 Elsner and the author constructed a leaping three-term recurrence relation from the original relation. Namely, for fixed positive integers $k$ and $0 \leq i<k$, they obtained a three-term relation concerning $z_{n}=Z_{k n+i}$.

For integers $a, l$ with $l \geq 1$ we define the determinant

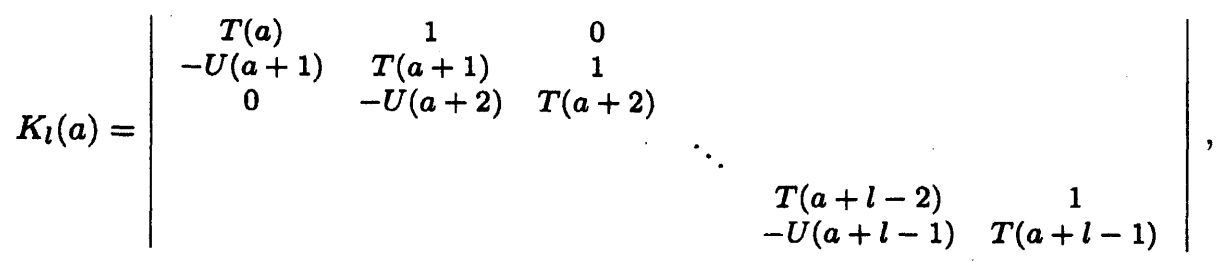

with $K_{0}(a)=1$. Let

$$
\Omega(M)=U(M-r) U(M-r+1) \ldots U(M-1)
$$

with $M=(n-1) r+i+2$. Then we have the following ([3, Theorem 2]).

Theorem 1 Given a three-term recurrence formula

$$
Z_{n}=T(n) Z_{n-1}+U(n) Z_{n-2} \quad(n \geq 2)
$$

with arbitrary initial values $Z_{0}, Z_{1}$ and two sequences of integers,

$$
\begin{aligned}
& (T(n))_{n \geq 0}=\left(a_{0}, a_{1}, a_{2}, \ldots, a_{\rho}, \overline{T_{1}(k), T_{2}(k), \ldots, T_{w}(k)}\right)_{k=1}^{\infty}, \\
& (U(n))_{n \geq 0}=\left(b_{0}, b_{1}, b_{2}, \ldots, b_{\rho}, \overline{U_{1}(k), U_{2}(k), \ldots, U_{w}(k)}\right)_{k=1}^{\infty},
\end{aligned}
$$

where $U(n) \neq 0$ for all $n \geq 0$, and $\rho \geq 0, w \geq 1$ are fixed integers. Then, for any integers $r$ and $i$ with $r \geq 2,0 \leq \rho \leq i<\rho+r$ and $n \geq 2$,

$$
\begin{gathered}
K_{r-1}(M-r) \cdot z_{n}-\left(K_{r-1}(M) K_{r}(M-r)+U(M) K_{r-1}(M-r) K_{r-2}(M+1)\right) \cdot z_{n-1} \\
+(-1)^{r} \Omega(M) K_{r-1}(M) \cdot z_{n-2}=0
\end{gathered}
$$

holds for $z_{n}=Z_{r n+i}$. For $T(a)>0$ and $U(a)>0$ for all $a>\rho$ one has $K_{r-1}(M) \neq 0$.

In particular, with $Z_{n}=q_{n}, q_{0}=1, q_{1}=a_{1}$ or $Z_{n}=p_{n}, p_{0}=a_{0}, p_{1}=a_{0} a_{1}+b_{1}$, this recurrence formula for $z_{n}$ is satisfied by the denominators $q_{r n+i}$ and numerators $p_{r n+i}$, respectively, of the convergents of a non-regular continued fraction

$$
\left[a_{0}+\frac{b_{1}}{a_{1}}+\frac{b_{2}}{a_{2}}+\cdots+\frac{b_{\rho}}{a_{\rho}}+\overline{\frac{U_{1}(k)}{T_{1}(k)}+\frac{U_{2}(k)}{T_{2}(k)}+\cdots+\frac{U_{w}(k)}{T_{w}(k)}}\right]_{k=1}^{\infty} .
$$

\footnotetext{
${ }^{1}$ This research was partially supported by the Grant-in-Aid for Scientific Research (C) (No. 18540006), the Japan Society for the Promotion of Science.
} 
In the case of regular continued fractions this result is reduced as follows.

Corollary 1 Given a three-term recurrence formula

$$
Z_{n}=T(n) Z_{n-1}+Z_{n-2} \quad(n \geq 2)
$$

with arbitrary initial values $Z_{0}, Z_{1}$ and a sequence of integers,

$$
(T(n))_{n \geq 0}=\left(a_{0}, a_{1}, a_{2}, \ldots, a_{\rho}, \overline{T_{1}(k), T_{2}(k), \ldots, T_{w}(k)}\right)_{k=1}^{\infty},
$$

where $\rho \geq 0$ and $w \geq 1$ are fixed integers. Then, for any integers $r$ and $i$ with $r \geq 2,0 \leq \rho \leq i<\rho+r$ and $n \geq 2$,

$$
\begin{gathered}
K_{r-1}(M-r) \cdot z_{n}-\left(K_{r-1}(M) K_{r}(M-r)+K_{r-1}(M-r) K_{r-2}(M+1)\right) \cdot z_{n-1} \\
+(-1)^{r} K_{r-1}(M) \cdot z_{n-2}=0
\end{gathered}
$$

holds for $z_{n}=Z_{r n+i}$. For $T(a)>0$ for all $a>\rho$ one has $K_{r-1}(M) \neq 0$.

In particular, with $Z_{n}=q_{n}, q_{0}=1, q_{1}=a_{1}$ or $Z_{n}=p_{n}, p_{0}=a_{0}, p_{1}=a_{0} a_{1}+1$, this recurrence formula for $z_{n}$ is satisfied by the denominators $q_{r n+i}$ and numerators $p_{r n+i}$, respectively, of the convergents of a regular continued fraction

$$
\left[a_{0} ; a_{1}, a_{2}, \ldots, a_{\rho}, \overline{T_{1}(k), T_{2}(k), \ldots, T_{w}(k)}\right]_{k=1}^{\infty}
$$

Three-term leaping recurrence relations which are entailed from continued fractions were studied in the case of $e$ by Elsner ([1]). Similar relations were also studied in the case of $e^{1 / s}(s \geq 2)$ by the author ([7], [8]). Such concepts were extended to the cases of more regular continued fractions and non-regular continued fractions ([2], [3]).

However, it is not easy to find an anologous result for linear four-term recurrence relations

$$
Z_{n}=U_{1}(n) Z_{n-1}+U_{2}(n) Z_{n-2}+U_{3}(n) Z_{n-3}
$$

where $U_{1}(n), U_{2}(n)$ and $U_{3}(n)$ are general sequences of integers. In this article we shall consider the leaping recurrence relations for four-term recurrence relations where $U_{1}(n)=a_{1}, U_{2}(n)=a_{2}$ and $U_{3}(n)=$ $a_{3}$ are integer constants. Then we can have the leaping relation

$$
Z_{n}=b_{1} Z_{n-k}+b_{2} Z_{n-2 k}+b_{3} Z_{n-3 k} \quad(n=3 k, 3 k+1,3 k+2, \ldots)
$$

for any leaping step $k$.

\section{Leaping convergents}

Let $\alpha$ be a real number. Continued fraction expansion of $\alpha$ is denoted by

$$
\alpha=\left[a_{0} ; a_{1}, a_{2}, \ldots\right]=a_{0}+\frac{1}{a_{1}+\frac{1}{a_{2}+\ldots}} .
$$

The $n$-th convergent is given by the irreducible rational number

$$
\frac{p_{n}}{q_{n}}=\left[a_{0} ; a_{1}, a_{2} \ldots, a_{n}\right]
$$

It is well-known that $p_{n}$ 's and $q_{n}$ 's satisfy the recurrence relations:

$$
\begin{array}{llll}
p_{n}=a_{n} p_{n-1}+p_{n-2} & (n \geq 0), & p_{-1}=1, & p_{-2}=0, \\
q_{n}=a_{n} q_{n-1}+q_{n-2} & (n \geq 0), & q_{-1}=1, & q_{-2}=0 .
\end{array}
$$


Leaping convergents of continued fractions are those of every $r$-th convergent of continued fractions;

$$
\frac{p_{i}}{q_{i}}, \quad \frac{p_{r+i}}{q_{r+i}}, \quad \frac{p_{2 r+i}}{q_{2 r+i}}, \ldots, \quad \frac{p_{r n+i}}{q_{r n+i}}, \ldots
$$

For example, consider

$$
e^{1 / s}=[1 ; \overline{(2 k-1) s-1,1,1}]_{k=1}^{\infty}=[1 ; s-1,1,1,3 s-1,1,1, \ldots] \quad(s \geq 2),
$$

then $p_{3 n}=2 s(2 n-1) p_{3 n-3}+p_{3 n-6}$ and $q_{3 n}=2 s(2 n-1) q_{3 n-3}+q_{3 n-6}(n \geq 2)([7])$.

\section{Three-term relations}

Three-term relations have been considered as in the continued fraction expansion of $e([1])$, as in that of $e^{1 / \bullet}(s \geq 2)([7],[8])$, as in that of the type $\left[1 ; \overline{T_{1}(k), T_{2}(k), T_{3}(k)}\right]_{k=1}^{\infty}([9],[10])$. Recently , three-term relations have been developed in the non-regular continued fractions $([2])$, and finally as in Theorem 1 here ([3]).

On the other direction, one can simplify the general theorem, entailing some classical results. If $T(n)=a_{1}, U(n)=a_{2}\left(a_{2} \neq 0\right)$ are integer constants in Theorem 1, we have the following.

Theorem 2 If the sequence $\left\{Z_{n}\right\}_{n}$ satisfies the three-term recurrence relation $Z_{n}=a_{1} Z_{n-1}+a_{2} Z_{n-2}$ $\left(a_{2} \neq 0\right)$, then for any positive integer $k$ we have

$$
Z_{n}=k \sum_{i=0}^{\lfloor k / 2\rfloor} \frac{(k-i-1) !}{i !(k-2 i) !} a_{1}^{k-2 i} a_{2}^{i} \cdot Z_{n-k}+(-1)^{k+1} a_{2}^{k} \cdot Z_{n-2 k} \quad(n \geq 2 k) .
$$

Moreover, if $a_{1}=a_{2}=1$, then the sequence $\left\{Z_{n}\right\}_{n}$ is called Fibonacci-type sequence. Moreover, if $Z_{0}=0$ and $Z_{1}=1$, then $\left\{Z_{n}\right\}_{n}$ is the Fibonacci sequence $\left\{F_{n}\right\}_{n}$.

Corollary 2 For any positive integer $k$ we have

$$
F_{n}=k \sum_{i=0}^{\lfloor k / 2\rfloor} \frac{(k-i-1) !}{i !(k-2 i) !} \cdot F_{n-k}-(-1)^{k} \cdot F_{n-2 k} \quad(n \geq 2 k) .
$$

If we put $k=2,3, \ldots, 10$, then we have

$$
\begin{aligned}
& F_{n}=3 F_{n-2}-F_{n-4}, \\
& F_{n}=4 F_{n-3}+F_{n-6}, \\
& F_{n}=7 F_{n-4}-F_{n-8}, \\
& F_{n}=11 F_{n-5}+F_{n-10}, \\
& F_{n}=18 F_{n-6}-F_{n-12}, \\
& F_{n}=29 F_{n-7}+F_{n-14}, \\
& F_{n}=47 F_{n-8}-F_{n-16}, \\
& F_{n}=76 F_{n-9}+F_{n-18}, \\
& F_{n}=123 F_{n-10}-F_{n-20} .
\end{aligned}
$$

There is a classical result corresponding to this corollary (Ruggles, 1963 [11, identity 105, p.92]):

$$
F_{n}=L_{k} F_{n-k}+(-1)^{k+1} F_{n-2 k},
$$


where $F_{n}$ and $L_{n}$ are Fibonacci number and Lucas numbers, respectively. Namely, they satisfy the three-term relations

$$
\begin{array}{llll}
F_{n}=F_{n-1}+F_{n-2} & (n \geq 2), & F_{0}=0, & F_{1}=1 \\
L_{n}=L_{n-1}+L_{n-2} & (n \geq 2), & L_{0}=2, & L_{1}=1 .
\end{array}
$$

Comparing (2) with (1), we have

\section{Corollary 3}

$$
L_{k}=k \sum_{i=0}^{\lfloor k / 2\rfloor} \frac{(k-i-1) !}{i !(k-2 i) !} \quad(k \geq 1) .
$$

Proof of Theorem 2. Set $K_{l}=K_{l}(c)$. Then, $\left\{K_{l}\right\}_{l \geq 0}$ satisfies the recurrence relation:

$$
K_{l}=a_{1} K_{l-1}+a_{2} K_{l-2} \quad(l \geq 2), \quad K_{0}=1, \quad K_{1}=a_{1} .
$$

Hence, for $l \geq 0$ we have

$$
K_{l}=\sum_{i=0}^{\lfloor l / 2\rfloor} \frac{(l-i) !}{i !(l-2 i) !} a_{1}^{l-2 i} a_{2}^{i} .
$$

Applying Theorem 1 with $\Omega(M)=a_{2}^{r}$, we have

$$
Z_{n}=\left(K_{r}+a_{2} K_{r-2}\right) \cdot Z_{n-1}-(-1)^{r} a_{2}^{r} \cdot Z_{n-2} .
$$

Since

$$
\begin{aligned}
K_{r}+a_{2} K_{r-2} & =\sum_{i=0}^{\lfloor r / 2\rfloor} \frac{(r-i) !}{i !(r-2 i) !} a_{1}^{r-2 i} a_{2}^{i}+\sum_{i=0}^{\lfloor r / 2\rfloor-1} \frac{(r-i-2) !}{i !(r-2 i-2) !} a_{1}^{r-2 i-2} a_{2}^{i+1} \\
& =r \sum_{i=0}^{\lfloor r / 2\rfloor} \frac{(r-i-1) !}{i !(r-2 i) !} a_{1}^{r-2 i} a_{2}^{i},
\end{aligned}
$$

we obtain the desired result.

\section{Four-term relations}

Consider the four-term recurrence relation

$$
Z_{n}=U_{1}(n) Z_{n-1}+U_{2}(n) Z_{n-2}+U_{3}(n) Z_{n-3}
$$

For the moment, the corresponding result to Theorem 1 has not been known. However, one can relax the conditions, in order to get some typical results. If $U_{1}(n)=a_{1}, U_{2}(n)=a_{2}, U_{3}(n)=a_{3}$ are constants for all $n$, we have the following four-term leaping recurrence relation.

Theorem 3 If the sequence $\left\{Z_{n}\right\}_{n}$ satisfies the four-term recurrence relation $Z_{n}=a_{1} Z_{n-1}+a_{2} Z_{n-2}+$ $a_{3} Z_{n-3}\left(a_{3} \neq 0\right)$, then for any positive integer $k$

$$
\begin{aligned}
Z_{n}=k & \sum_{j=0}^{\lfloor k / 3\rfloor} \sum_{i=0}^{\lfloor(k-3 j) / 2\rfloor} \frac{(k-i-2 j-1) !}{i ! j !(k-2 i-3 j) !} a_{1}^{k-2 i-3 j} a_{2}^{i} a_{3}^{j} \cdot Z_{n-k} \\
& -k \sum_{j=0}^{\lfloor k / 3\rfloor} \sum_{i=0}^{\lfloor(k-3 j) / 2\rfloor}(-1)^{k+i+j} \frac{(k-i-2 j-1) !}{i ! j !(k-2 i-3 j) !} a_{1}^{i} a_{2}^{k-2 i-3 j} a_{3}^{i+2 j} \cdot Z_{n-2 k}+a_{3}^{k} \cdot Z_{n-3 k}
\end{aligned}
$$


In $2001 \mathrm{~F}$. T. Howard obtained a similar result ([5]):

where $J_{n}$ satisfies

$$
Z_{n}=J_{k} Z_{n-k}-a_{3}^{k} J_{-k} Z_{n-2 k}+a_{3}^{k} Z_{n-3 k}
$$

$$
\begin{aligned}
& J_{n}=a_{1} J_{n-1}+a_{2} J_{n-2}+a_{3} J_{n-3} \quad(n \geq 3), \\
& J_{0}=3, \quad J_{1}=a_{1}, \quad J_{2}=a_{1}^{2}+2 a_{2} .
\end{aligned}
$$

$J_{-n}(n=1,2, \ldots)$ are determined by

$$
J_{-n}=\frac{1}{a_{3}}\left(J_{-n+3}-a_{1} J_{-n+2}-a_{2} J_{-n+1}\right) \quad(n \geq 1) .
$$

Comparing (3) with Theorem 3, we obtain

\section{Corollary 4}

$$
\begin{aligned}
J_{k} & =k \sum_{j=0}^{\lfloor k / 3\rfloor} \sum_{i=0}^{\lfloor(k-3 j) / 2\rfloor} \frac{(k-i-2 j-1) !}{i ! j !(k-2 i-3 j) !} a_{1}^{k-2 i-3 j} a_{2}^{i} a_{3}^{j}, \\
J_{-k} & =k \sum_{j=0}^{\lfloor k / 3\rfloor} \sum_{i=0}^{\lfloor(k-3 j) / 2\rfloor}(-1)^{k+i+j} \frac{(k-i-2 j-1) !}{i ! j !(k-2 i-3 j) !} a_{1}^{i} a_{2}^{k-2 i-3 j} a_{3}^{-k+i+2 j}
\end{aligned}
$$

In the case of $k=12$,

$$
\begin{gathered}
Z_{n}-\left(a_{1}^{12}+12 a_{1}^{10} a_{2}+54 a_{1}^{8} a_{2}^{2}+112 a_{1}^{6} a_{2}^{3}+105 a_{1}^{4} a_{2}^{4}+36 a_{1}^{2} a_{2}^{5}+2 a_{2}^{6}\right. \\
+12 a_{1}^{9} a_{3}+96 a_{1}^{7} a_{2} a_{3}+252 a_{1}^{5} a_{2}^{2} a_{3}+240 a_{1}^{3} a_{2}^{3} a_{3}+60 a_{1} a_{2}^{4} a_{3}+42 a_{1}^{6} a_{3}^{2} \\
\left.+180 a_{1}^{4} a_{2} a_{3}^{2}+180 a_{1}^{2} a_{2}^{2} a_{3}^{2}+24 a_{2}^{3} a_{3}^{2}+40 a_{1}^{3} a_{3}^{3}+48 a_{1} a_{2} a_{3}^{3}+3 a_{3}^{4}\right) Z_{n-12} \\
+\left(a_{2}^{12}-12 a_{1} a_{2}^{10} a_{3}+54 a_{1}^{2} a_{2}^{8} a_{3}^{2}-12 a_{2}^{9} a_{3}^{2}-112 a_{1}^{3} a_{2}^{6} a_{3}^{3}+96 a_{1} a_{2}^{7} a_{3}^{3}\right. \\
+105 a_{1}^{4} a_{2}^{4} a_{3}^{4}-252 a_{1}^{2} a_{2}^{5} a_{3}^{4}+42 a_{2}^{6} a_{3}^{4}-36 a_{1}^{5} a_{2}^{2} a_{3}^{5}+240 a_{1}^{3} a_{2}^{3} a_{3}^{5}-180 a_{1} a_{2}^{4} a_{3}^{5} \\
+2 a_{1}^{6} a_{3}^{6}-60 a_{1}^{4} a_{2} a_{3}^{6}+180 a_{1}^{2} a_{2}^{2} a_{3}^{6}-40 a_{2}^{3} a_{3}^{6}-24 a_{1}^{3} a_{3}^{7} \\
\left.+48 a_{1} a_{2} a_{3}^{7}+3 a_{3}^{8}\right) Z_{n-24}-a_{3}^{12} Z_{n-36}=0 .
\end{gathered}
$$

Put $a_{1}=a_{2}=a_{3}=1$. Then the four-term recurrence relation $T_{n}=T_{n-1}+T_{n-2}+T_{n-3}$ yields Tribonacci numbers $\left\{T_{n}\right\}_{n \geq 0}$. If $\left(T_{0}=0,\right) T_{1}=T_{2}=1$ and $T_{3}=2$, then the Tribonacci sequence is given by

$$
1,1,2,4,7,13,24,44,81,149,274,504,927,1705,3136,5768,10609,19513,35890,66012,121415, \ldots .
$$

([11, p.527], [12, A000073]). Putting $k=2,3, \ldots, 10$ in Theorem 3, we have

$$
\begin{aligned}
& T_{n}=3 T_{n-2}+T_{n-4}+T_{n-6}, \\
& T_{n}=7 T_{n-3}-5 T_{n-6}+T_{n-9}, \\
& T_{n}=11 T_{n-4}+5 T_{n-8}+T_{n-12}, \\
& T_{n}=21 T_{n-5}+T_{n-10}+T_{n-15}, \\
& T_{n}=39 T_{n-6}-11 T_{n-12}+T_{n-18}, \\
& T_{n}=71 T_{n-7}+15 T_{n-14}+T_{n-21}, \\
& T_{n}=131 T_{n-8}-3 T_{n-16}+T_{n-24}, \\
& T_{n}=241 T_{n-9}-23 T_{n-18}+T_{n-27}, \\
& T_{n}=443 T_{n-10}+41 T_{n-20}+T_{n-30} .
\end{aligned}
$$




\section{Five-term relations}

Consider the sequence $\left\{Z_{n}\right\}_{n}$ satisfying the five-term recurrence relation $Z_{n}=a_{1} Z_{n-1}+a_{2} Z_{n-2}+$ $a_{3} Z_{n-3}+a_{4} Z_{n-4}\left(a_{4} \neq 0\right)$. Then how can we determine the integer constants $b_{1}, b_{2}, b_{3}, b_{4}$, satisfying

$$
Z_{n}=b_{1} Z_{n-k}+b_{2} Z_{n-2 k}+b_{3} Z_{n-3 k}+b_{4} Z_{n-4 k}
$$

for any positive integer $k(1<k<n / 4)$ ?

In the case of $k=5$

$$
\begin{aligned}
Z_{n}=\left(a_{1}^{5}+5 a_{1}^{3} a_{2}+\right. & \left.5 a_{1} a_{2}^{2}+5 a_{1}^{2} a_{3}+5 a_{2} a_{3}+5 a_{1} a_{4}\right) Z_{n-5} \\
+ & \left(a_{2}^{5}-5 a_{1} a_{2}^{3} a_{3}+5 a_{1}^{2} a_{2} a_{3}^{2}-5 a_{2}^{2} a_{3}^{2}+5 a_{1} a_{3}^{3}+5 a_{1}^{2} a_{2}^{2} a_{4}+5 a_{2}^{3} a_{4}\right. \\
& \left.-5 a_{1}^{3} a_{3} a_{4}+5 a_{1} a_{2} a_{3} a_{4}+5 a_{3}^{2} a_{4}-5 a_{1}^{2} a_{4}^{2}+5 a_{2} a_{4}^{2}\right) Z_{n-10} \\
& +\left(a_{3}^{5}-5 a_{2} a_{3}^{3} a_{4}+5 a_{2}^{2} a_{3} a_{4}^{2}+5 a_{1} a_{3}^{2} a_{4}^{2}-5 a_{1} a_{2} a_{4}^{3}+5 a_{3} a_{4}^{3}\right) Z_{n-15}+a_{4}^{5} Z_{n-20} .
\end{aligned}
$$

In the case of $k=6$,

$$
\begin{aligned}
Z_{n}=\left(a_{1}^{6}+6 a_{1}^{4} a_{2}+9 a_{1}^{2} a_{2}^{2}+2 a_{2}^{3}+6 a_{1}^{3} a_{3}+12 a_{1} a_{2} a_{3}+3 a_{3}^{2}+6 a_{1}^{2} a_{4}+6 a_{2} a_{4}\right) Z_{n-6} \\
+\left(-a_{2}^{6}+6 a_{1} a_{2}^{4} a_{3}-9 a_{1}^{2} a_{2}^{2} a_{3}^{2}+6 a_{2}^{3} a_{3}^{2}+2 a_{1}^{3} a_{3}^{3}-12 a_{1} a_{2} a_{3}^{3}-3 a_{3}^{4}-6 a_{1}^{2} a_{2}^{3} a_{4}\right. \\
\left.-6 a_{2}^{4} a_{4}+12 a_{1}^{3} a_{2} a_{3} a_{4}+18 a_{1}^{2} a_{3}^{2} a_{4}-3 a_{1}^{4} a_{4}^{2}-9 a_{2}^{2} a_{4}^{2}+18 a_{1} a_{3} a_{4}^{2}+2 a_{4}^{3}\right) Z_{n-12} \\
+\left(a_{3}^{6}-6 a_{2} a_{3}^{4} a_{4}+9 a_{2}^{2} a_{3}^{2} a_{4}^{2}+6 a_{1} a_{3}^{3} a_{4}^{2}-2 a_{2}^{3} a_{4}^{3}-12 a_{1} a_{2} a_{3} a_{4}^{3}+6 a_{3}^{2} a_{4}^{3}+3 a_{1}^{2} a_{4}^{4}-6 a_{2} a_{4}^{4}\right) Z_{n-18} \\
-a_{4}^{6} Z_{n-24} .
\end{aligned}
$$

Tetranacci Numbers $\left\{F_{k}^{(4)}\right\}_{k \geq 1}$ are the $n=4$ case of the Fibonacci $n$-step Numbers, defined by $F_{k}^{(4)}=F_{k-1}^{(4)}+F_{k-2}^{(4)}+F_{k-3}^{(4)}+F_{k-4}^{(4)}(k \geq 5)$ with $F_{1}^{(4)}=F_{2}^{(4)}=1, F_{3}^{(4)}=2$ and $F_{4}^{(4)}=4$. The first terms are

$1,1,2,4,8,15,29,56,108,208,401,773,1490,2872,5536,10671,20569,39648,76424,147312$, 283953, 547337, 1055026, 2033628, 3919944, 7555935, 14564533, 28074040, 54114452, 104308960, . .

([12, A000078]). They satisfy the recurrence relations:

$$
\begin{aligned}
& F_{k}^{(4)}=3 F_{k-2}^{(4)}+3 F_{k-4}^{(4)}-F_{k-6}^{(4)}-F_{k-8}^{(4)}, \\
& F_{k}^{(4)}=7 F_{k-3}^{(4)}+F_{k-6}^{(4)}+F_{k-8}^{(4)}+F_{k-12}^{(4)}, \\
& F_{k}^{(4)}=15 F_{k-4}^{(4)}-17 F_{k-8}^{(4)}+7 F_{k-12}^{(4)}-F_{k-16}^{(4)}, \\
& F_{k}^{(4)}=26 F_{k-5}^{(4)}+16 F_{k-10}^{(4)}+6 F_{k-15}^{(4)}+F_{k-20}^{(4)}, \\
& F_{k}^{(4)}=51 F_{k-6}^{(4)}+15 F_{k-12}^{(4)}-F_{k-18}^{(4)}-F_{k-24}^{(4)}, \\
& F_{k}^{(4)}=99 F_{k-7}^{(4)}-13 F_{k-14}^{(4)}+F_{k-21}^{(4)}+F_{k-28}^{(4)}, \\
& F_{k}^{(4)}=191 F_{k-8}^{(4)}-81 F_{k-16}^{(4)}+15 F_{k-24}^{(4)}-F_{k-32}^{(4)}, \\
& F_{k}^{(4)}=367 F_{k-9}^{(4)}+127 F_{k-18}^{(4)}+19 F_{k-27}^{(4)}+F_{k-36}^{(4)}, \\
& F_{k}^{(4)}=708 F_{k-10}^{(4)}+58 F_{k-20}^{(4)}+4 F_{k-30}^{(4)}-F_{k-40}^{(4)}
\end{aligned}
$$

$b_{1}, b_{3}$ and $b_{4}$ are calculated as follows. 


\section{Theorem 4}

$$
\begin{aligned}
& b_{1}=k \sum_{\kappa=0}^{\lfloor k / 4\rfloor} \sum_{j=0}^{\lfloor(k-4 \kappa) / 3\rfloor} \sum_{i=0}^{\lfloor(k-3 j-4 \kappa) / 2\rfloor} \frac{(k-i-2 j-3 \kappa-1) !}{i ! j ! \kappa !(k-2 i-3 j-4 \kappa) !} a_{1}^{k-2 i-3 j-4 \kappa} a_{2}^{i} a_{3}^{j} a_{4}^{\kappa}, \\
& b_{3}=k \sum_{\kappa=0}^{\lfloor k / 4\rfloor} \sum_{j=0}^{\lfloor(k-4 \kappa) / 3\rfloor} \sum_{i=0}^{\lfloor(k-3 j-4 \kappa) / 2\rfloor}(-1)^{i} \frac{(k-i-2 j-3 \kappa-1) !}{i ! j ! \kappa !(k-2 i-3 j-4 \kappa) !} a_{1}^{j} a_{2}^{i} a_{3}^{k-2 i-3 j-4 \kappa} a_{4}^{i+2 j+3 \kappa}, \\
& b_{4}=(-1)^{k-1} a_{4}^{k} .
\end{aligned}
$$

However, it is not easy to find an explicit form of $b_{2}$. This shall be discussed in the next section.

In 2005 Latushkin and Ushakov ([6]) obtained a different form of five-term leaping relations.

$$
Z_{n}=H_{k} Z_{n-k}+\frac{H_{2 k}-H_{k}^{2}}{2} Z_{n-2 k}+\left(-a_{4}\right)^{k} H_{-k} Z_{n-3 k}-\left(-a_{4}\right)^{k} Z_{n-4 k}
$$

where

$$
H_{n}=x_{1}^{n}+x_{2}^{n}+x_{3}^{n}+x_{4}^{n} \quad(n \in \mathbb{Z})
$$

and $x_{1}, x_{2}, x_{3}$ and $x_{4}$ are the complex roots (including multiple roots) of the equation $x^{4}-a_{1} x^{3}-a_{2} x^{2}-$ $a_{3} x-a_{4}=0$. On the other hand, the sequence $\left\{H_{n}\right\}_{n}$ satisfies the recurrence relation:

$$
H_{n}=a_{1} H_{n-1}+a_{2} H_{n-2}+a_{3} H_{n-3}+a_{4} H_{n-4} \quad(n \in \mathbb{Z}) \text {. }
$$

The initial values are determined by

$$
\begin{aligned}
& H_{0}=4 \\
& H_{1}=a_{1} \\
& H_{2}=a_{1} H_{1}+2 a_{2}=a_{1}^{2}+2 a_{2} \\
& H_{3}=a_{1} H_{2}+a_{2} H_{1}+3 a_{3}=a_{1}^{3}+3 a_{1} a_{2}+3 a_{3} \\
& H_{4}=a_{1} H_{3}+a_{2} H_{2}+a_{3} H_{1}+4 a_{4}=a_{1}^{4}+4 a_{1} a_{2}+4 a_{1} a_{3}+2 a_{2}^{2}+4 a_{4} .
\end{aligned}
$$

Comparing their results (4) with ours in Theorem 4, we get the following.

\section{Corollary 5}

$$
\begin{aligned}
H_{k} & =k \sum_{\kappa=0}^{\lfloor k / 4\rfloor} \sum_{j=0}^{\lfloor(k-4 \kappa) / 3\rfloor} \sum_{i=0}^{\lfloor(k-3 j-4 \kappa) / 2\rfloor} \frac{(k-i-2 j-3 \kappa-1) !}{i ! j ! \kappa !(k-2 i-3 j-4 \kappa) !} a_{1}^{k-2 i-3 j-4 \kappa} a_{2}^{i} a_{3}^{j} a_{4}^{\kappa} \\
H_{-k} & =k \sum_{\kappa=0}^{\lfloor k / 4\rfloor} \sum_{j=0}^{\lfloor(k-4 \kappa) / 3\rfloor} \sum_{i=0}^{\lfloor(k-3 j-4 \kappa) / 2\rfloor}(-1)^{i+k} \frac{(k-i-2 j-3 \kappa-1) !}{i ! j ! \kappa !(k-2 i-3 j-4 \kappa) !} a_{1}^{j} a_{2}^{i} a_{3}^{k-2 i-3 j-4 \kappa} a_{4}^{-k+i+2 j+3 \kappa}
\end{aligned}
$$

Pentanacci Numbers $\left\{F_{k}^{(5)}\right\}_{k \geq 1}$ are the $n=5$ case of the Fibonacci $n$-step Numbers, defined by $F_{k}^{(5)}=F_{k-1}^{(5)}+F_{k-2}^{(5)}+F_{k-3}^{(5)}+F_{k-4}^{(5)}+F_{k-5}^{(5)}(k \geq 6)$ with $F_{1}^{(5)}=F_{2}^{(5)}=1, F_{3}^{(5)}=2, F_{4}^{(5)}=4$ and $F_{5}^{(5)}=8$ The first terms are 
([12, A001591]). They satisfy the recurrence relations:

$$
\begin{aligned}
& F_{k}^{(5)}=3 F_{k-2}^{(5)}+3 F_{k-4}^{(5)}+F_{k-6}^{(5)}+F_{k-8}^{(5)}+F_{k-10}^{(5)} \\
& F_{k}^{(5)}=7 F_{k-3}^{(5)}+4 F_{k-6}^{(5)}+4 F_{k-9}^{(5)}+F_{k-12}^{(5)}+F_{k-15}^{(5)} \\
& F_{k}^{(5)}=15 F_{k-4}^{(5)}-F_{k-8}^{(5)}+F_{k-12}^{(5)}+F_{k-16}^{(5)}+F_{k-20}^{(5)} \\
& F_{k}^{(5)}=31 F_{k-5}^{(5)}-49 F_{k-10}^{(5)}+31 F_{k-15}^{(5)}-9 F_{k-20}^{(5)}+F_{k-25}^{(5)}, \\
& F_{k}^{(5)}=57 F_{k-6}^{(5)}+42 F_{k-12}^{(5)}+22 F_{k-18}^{(5)}+7 F_{k-24}^{(5)}+F_{k-30}^{(5)} \\
& F_{k}^{(5)}=113 F_{k-7}^{(5)}+57 F_{k-14}^{(5)}+F_{k-21}^{(5)}+F_{k-28}^{(5)}+F_{k-35}^{(5)} \\
& F_{k}^{(5)}=223 F_{k-8}^{(5)}+31 F_{k-16}^{(5)}+33 F_{k-24}^{(5)}+F_{k-32}^{(5)}+F_{k-40}^{(5)}, \\
& F_{k}^{(5)}=439 F_{k-9}^{(5)}-140 F_{k-18}^{(5)}+4 F_{k-27}^{(5)}+F_{k-36}^{(5)}+F_{k-45}^{(5)}, \\
& F_{k}^{(5)}=863 F_{k-10}^{(5)}-497 F_{k-20}^{(5)}+141 F_{k-30}^{(5)}-19 F_{k-40}^{(5)}+F_{k-30}^{(5)} .
\end{aligned}
$$

\section{A form of $b_{2}$ in five-term leaping relations}

An explicit form of $b_{2}$ has not been known yet. Instead, there is a way to express $b_{2}$ by matrices.

$$
\begin{aligned}
b_{2}= & a_{2} \Lambda_{k-1}-2 a_{3} \Phi_{k-1}+3 a_{4} \Psi_{k-1} \\
& -a_{3} \Phi_{k-1}+2 a_{4} \Lambda_{k-2}-a_{1} a_{3} \Lambda_{k-2}+2 a_{2} a_{4} \Lambda_{k-3}-3 a_{4}^{2} \Lambda_{k-4},
\end{aligned}
$$

where

$$
\begin{aligned}
& \Lambda_{n}=\left|\begin{array}{cccccccc}
-a_{2} & -a_{1} & 1 & 0 & & & & \\
-a_{3} & -a_{2} & -a_{1} & 1 & & & & \\
-a_{4} & -a_{3} & -a_{2} & -a_{1} & & & & \\
0 & & & \ddots & & & & \\
& & & & -a_{2} & -a_{1} & 1 & 0 \\
& & & & -a_{3} & -a_{2} & -a_{1} & 1 \\
& & & & -a_{4} & -a_{3} & -a_{2} & -a_{1} \\
& & & & & -a_{4} & -a_{3} & -a_{2}
\end{array}\right|, \\
& \Phi_{n}=\left|\begin{array}{cccccccc}
-a_{1} & 1 & 0 & & & & & \\
-a_{3} & -a_{2} & -a_{1} & 1 & 0 & & & \\
-a_{4} & -a_{3} & -a_{2} & -a_{1} & 1 & & & \\
0 & & & \ddots & & & & \\
& & & & -a_{2} & -a_{1} & 1 & 0 \\
& & & & -a_{3} & -a_{2} & -a_{1} & 1 \\
& & & & -a_{4} & -a_{3} & -a_{2} & -a_{1} \\
& & & & 0 & -a_{4} & -a_{3} & -a_{2}
\end{array}\right|, \\
& \Psi_{n}=\left|\begin{array}{cccccccc}
-a_{1} & 1 & 0 & & & & & \\
-a_{2} & -a_{1} & 1 & 0 & & & & \\
-a_{4} & -a_{3} & -a_{2} & -a_{1} & 1 & & & \\
0 & & & \ddots & & & & \\
& & & & -a_{2} & -a_{1} & 1 & 0 \\
& & & & -a_{3} & -a_{2} & -a_{1} & 1 \\
& & & & -a_{4} & -a_{3} & -a_{2} & -a_{1} \\
& & & & 0 & -a_{4} & -a_{3} & -a_{2}
\end{array}\right| .
\end{aligned}
$$


Notice that

$$
\begin{aligned}
& \Lambda_{n}=-a_{2} \Lambda_{n-1}+a_{3} \Phi_{n-1}-a_{4} \Psi_{n-1}, \\
& \Phi_{n}=-a_{1} \Lambda_{n-1}+a_{3} \Lambda_{n-2}-a_{4} \Phi_{n-2}, \\
& \Psi_{n}=-a_{1} \Phi_{n-1}+a_{2} \Lambda_{n-2}-a_{4} \Lambda_{n-3} .
\end{aligned}
$$

$b_{2}$ may be expanded as follows.

$$
\begin{aligned}
b_{2}= & (-1)^{k-1} k\left(a_{2}^{k}-\left(a_{1} a_{3}-a_{4}\right) a_{2}^{k-2}-\left(a_{3}^{2}-a_{1}^{2} a_{4}\right) a_{2}^{k-3}\right. \\
+ & \left(\frac{(k-3) !}{2 !(k-4) !} a_{1}^{2} a_{3}^{2}-\frac{(k-4) !}{(k-4) !}(k-6) a_{1} a_{3} a_{4}+\frac{k-3}{2} a_{4}^{2}\right) a_{2}^{k-4} \\
+ & \left(a_{3}^{2}-a_{1}^{2} a_{4}\right)\left(\frac{(k-4) !}{(k-5) !} a_{1} a_{3}-(k-6) a_{4}\right) a_{2}^{k-5} \\
& -\left(\frac{(k-4) !}{3 !(k-6) !} a_{1}^{3} a_{3}^{3}-\frac{(k-5) !}{2 !(k-6) !}(k-12) a_{1}^{2} a_{3}^{2} a_{4}+\frac{k^{2}-15 k+60}{2} a_{1} a_{3} a_{4}^{2}\right. \\
& \left.-\frac{(k-4) !}{3 !(k-6) !} a_{4}^{3}-\frac{k-5}{2}\left(a_{1}^{4} a_{4}^{2}+a_{3}^{4}\right)\right) a_{2}^{k-6} \\
- & \left(a_{3}^{2}-a_{1}^{2} a_{4}\right)\left(\frac{(k-5) !}{2 !(k-7) !} a_{1}^{2} a_{3}^{2}-\frac{(k-6) !}{(k-7) !}(k-10) a_{1} a_{3} a_{4}+\frac{k^{2}-15 k+60}{2} a_{4}^{2}\right) a_{2}^{k-7} \\
+ & \left(\frac{(k-5) !}{4 !(k-8) !} a_{1}^{4} a_{3}^{4}-\frac{(k-6) !}{3 !(k-8) !}(k-20) a_{1}^{3} a_{3}^{3} a_{4}\right. \\
+ & \frac{(k-7) !}{2 ! 2 !(k-8) !}(k-12)(k-15) a_{1}^{2} a_{3}^{2} a_{4}^{2}-\frac{(k-10)\left(k^{2}-17 k+84\right)}{3 !} a_{1} a_{3} a_{4}^{3} \\
+ & \left.\frac{(k-5) !}{4 !(k-8) !} a_{4}^{4}-\frac{k-7}{2}\left(a_{1}^{4} a_{4}^{2}+a_{3}^{4}\right)\left((k-6) a_{1} a_{3}-(k-10) a_{4}\right)\right) a_{2}^{k-8} \\
+ & \left(( a _ { 3 } ^ { 2 } - a _ { 1 } ^ { 2 } a _ { 4 } ) \left(\frac{(k-6) !}{3 !(k-9) !} a_{1}^{3} a_{3}^{3}-\frac{(k-7) !}{2 !(k-9) !}(k-15) a_{1}^{2} a_{3}^{2} a_{4}\right.\right. \\
& \left.+\frac{(k-8) !}{2 !(k-9) !}\left(k^{2}-23 k+140\right) a_{1} a_{3} a_{4}^{2}-\frac{(k-9) !}{3 !(k-9) !}(k-10)\left(k^{2}-17 k+84\right) a_{4}^{3}\right) \\
& \left.-\left(a_{3}^{6}-a_{1}^{6} a_{4}^{3}\right) \frac{(k-7) !}{3 !(k-9) !}\right) a_{2}^{k-9} \\
& +\cdots)
\end{aligned}
$$

However, its simplified form has not been known.

\section{$7 \quad(s+1)$-term recurrence relations}

We may extend terms to five, six, seven, and so on. In 1999 Howard got a general term leaping relation ([4]). Young also found a different form ([13]). This result holds for more-term recurrence relations, but it is not so useful practically in order to obtain an explicit form for any given $s$.

If the sequence $\left\{Z_{n}\right\}_{n \geq 0}$ satisfies the relation

$$
Z_{n}=a_{1} Z_{n-1}+a_{2} Z_{n-2}+\cdots+a_{s} Z_{n-s} \quad\left(a_{s} \neq 0\right),
$$

where $Z_{0}, Z_{1}, \ldots, Z_{s-1}$ are arbitrary initial values, then we have

$$
Z_{r n+i}=c_{r, r} Z_{r(n-1)+i}-c_{r, 2 r} Z_{r(n-2)+i}+\cdots+(-1)^{s-1} c_{r, s r} Z_{r(n-s)+i},
$$


where $c_{r, r}, c_{r, 2 r}, \ldots, c_{r, s r}$ are determined by

$$
\prod_{\nu=0}^{r-1}\left(1-a_{1}\left(\zeta^{\nu} x\right)-a_{2}\left(\zeta^{\nu} x\right)^{2}-\cdots-a_{s}\left(\zeta^{\nu} x\right)^{s}\right)=1-c_{r, r} x^{r}+c_{r, 2 r} x^{2 r}-\cdots+(-1)^{s} c_{r, s r} x^{s r},
$$

where $\zeta$ is a primitive $r$-th root of unity.

As straight generalization of our theorems 2, 3, 4, we obtain the following .

Theorem 5 If

$$
Z_{n}=b_{1} Z_{n-k}+b_{2} Z_{n-2 k}+\cdots+b_{s-1} Z_{n-(s-1) k}+b_{s} Z_{n-s k}
$$

then

$$
\begin{aligned}
& b_{1}=k \sum_{\substack{2 i_{1}+3 i_{2}+\cdots+b i_{-1} \leq k \\
i_{1}, i_{2} \ldots, \ldots, i_{s}-1 \geq 0}} \frac{\left(k-i_{1}-2 i_{2}-\cdots-(s-1) i_{s-1}-1\right) !}{i_{1} ! i_{2} ! \ldots i_{s-1} !\left(k-2 i_{1}-3 i_{2}-\cdots-s i_{s-1}\right) !} \\
& \times a_{1}^{k-2 i_{1}-3 i_{2}-\cdots-s i_{o-1}} a_{2}^{i_{1}} a_{3}^{i_{2}} \ldots a_{s}^{i_{s-1}}, \\
& b_{s-1}=k \sum_{\substack{2 i_{1}+8 i_{2}+\cdots+i_{s-1} \leq k \\
i_{1}, i_{2}, \ldots, i_{s}-1 \geq 0}}(-1)^{I-1} \frac{\left(k-i_{1}-2 i_{2}-\cdots-(s-1) i_{s-1}-1\right) !}{i_{1} ! i_{2} ! \ldots i_{o-1} !\left(k-2 i_{1}-3 i_{2}-\cdots-s i_{s-1}\right) !} \\
& \times a_{1}^{i_{s-2}} a_{2}^{i_{o-3}} \ldots a_{s-2}^{i_{1}} a_{s-1}^{n-2 i_{1}-3 i_{2}-\cdots-s i_{o-1}} a_{s}^{i_{1}+2 i_{2}+\cdots+(\bullet-1) i_{\Delta-1},}
\end{aligned}
$$

where

$$
I= \begin{cases}i_{1}+i_{3}+\cdots+i_{s-2}+i_{s-1} & \text { if } s \text { is odd } \\ i_{1}+i_{3}+\cdots+i_{s-3} & \text { if } s \text { is even }\end{cases}
$$

and

$$
b_{s}= \begin{cases}a_{s}^{k} & \text { if } s \text { is odd } \\ (-1)^{k-1} a_{s}^{k} & \text { if } s \text { is even. }\end{cases}
$$

\section{Periodicity}

In [2, Theorem 3] a result about periodicity of three-term leaping relations is obtained.

Theorem 6 Given a three-term recurrence formula

$$
Z_{n}=T(n) Z_{n-1}+U(n) Z_{n-2} \quad(n \geq 2)
$$

with arbitrary initial values $Z_{0}, Z_{1}$ and two sequences of integers $(T(n))_{n \geq 0}$ and $(U(n))_{n \geq 0}$, which both are (ultimately) periodic modulo $m$ with periods of length $r$, say

$$
\begin{aligned}
& (T(n) \bmod m)_{n \geq 0}=\left(a_{0}, a_{1}, a_{2}, \ldots, a_{\rho}, \overline{T_{1}, T_{2}, \ldots, T_{r}}\right), \\
& (U(n) \bmod m)_{n \geq 0}=\left(b_{0}, b_{1}, b_{2}, \ldots, b_{\rho}, \overline{U_{1}, U_{2}, \ldots, U_{r}}\right) .
\end{aligned}
$$

Then, the sequence $(Z(n))_{n>0}$ is (ultimately) periodic modulo $m$. If $\rho \in\{0,1\}$ and $U(n)=1$ for all $n \geq \rho$, then the sequence $(Z(n))_{n \geq 0}$ is periodic modulo $m$.

This result can be extended to the case of any term leaping relations.

Theorem 7 Given a $(s+1)$-term recurrence formula $Z_{n}=T_{1}(n) Z_{n-1}+T_{2}(n) Z_{n-2}+\cdots+T_{s}(n) Z_{n-s}(n \geq$ 2) with arbitrary initial values $Z_{0}, Z_{1}, \ldots, Z_{\text {s }}$ and $s$ sequences of integers $\left(T_{j}(n)\right)_{n \geq 0}(j=1,2, \ldots, s)$, which all are (ultimately) periodic modulo $m$ with periods of length $r$, then, the sequence $(Z(n))_{n \geq 0}$ is (ultimately) periodic modulo $m$. 


\section{References}

[1] C. Elsner, On arithmetic properties of the convergents of Euler's number, Colloq. Math. 79 (1999), 133-145.

[2] C. Elsner and T. Komatsu, A recurrence formula for leaping convergents of non-regular continued fractions, Linear Algebra Appl. 428 (2008) 824-833.

[3] C. Elsner and T. Komatsu, On the residue classes of integer sequences satisfying a linear three term recurrence formula, Linear Algebra Appl. 429 (2008) 933-947.

[4] F. T. Howard, Generalizations of a Fibonacci identity, in Applications of Fibonacci numbers 8, ed. by F. T. Howard, Dordrecht, Kluwer, 1999, pp. 201-211.

[5] F. T. Howard, A tribonacci identity, Fibonacci Quart. 39 (2001) 352-358.

[6] Y. Latushkin and V. Ushakov, A representation of regular subsequences of recurrent sequences, Fibonacci Quart. 43 (2005) 70-84.

[7] T. Komatsu, Recurrence relations of the leaping convergents, JP J. Algebra Number Theory Appl. 3 (2003), 447-459.

[8] T. Komatsu, Arithmetical properties of the leaping convergents of $e^{1 / s}$, Tokyo J. Math. 27 (2004), 1-12.

[9] T. Komatsu, Some combinatorial properties of the leaping convergents, in B. M. Landman, M. B. Nathanson, J. Nešetril, R. J. Nowakowski, and C. Pomerance (Eds.), Combinatorial Number Theory, Proceedings of the Integers Conference 2005 in Celebration of the 70th Birthday of Ronald Graham, Carrollton, Georgia, USA, October 27-30, 2005, Walter de Gruyter, 2007, 315-325.

[10] T. Komatsu, Some combinatorial properties of the leaping convergents, II, Applications of Fibonacci Numbers, Proceedings of the 12th International Conference on Fibonacci Numbers and their Applications, (accepted for publication).

[11] T. Koshy, Fibonacci and Lucas numbers with applications, John Wiley \& Sons, 2001.

[12] N. J. A. Sloane, The On-Line Encyclopedia of Integer Sequences, published electronically at www.research.att.com/ njas/sequences.

[13] P. T. Young, On lacunary recurrences, Fibonacci Quart. 41 (2003) 41-47.

Graduate School of Science and Technology

Hirosaki University, Hirosaki, 036-8561, Japan

komatsuecc.hirosaki-u.ac.jp 